\title{
Anatomical and Functional Brain Imaging in Childhood ADHD: Update 2013
}

\author{
Marco Angriman • Anita Beggiato • Samuele Cortese
}

Published online: 19 December 2013

(C) Springer International Publishing AG 2013

\begin{abstract}
Neuroimaging studies of attention-deficit/hyperactivity disorder (ADHD) are continuously adding to our knowledge on the neurobiology of this condition. Here, we update previous narrative and systematic reviews on the neuroimaging of ADHD, focusing on studies published in 2013. In order to comprehensively retrieve all the published studies, we searched a broad range of electronic databases (PubMed, Ovid PsychInfo, Ovide Medline, Web of Science, and EMBASE). We critically overview anatomical magnetic resonance imaging (MRI) and diffusion tensor imaging (DTI) studies, as well as functional MRI and resting state MRI studies. We also included studies aimed at elucidating the mechanism of action of ADHD drugs. We conclude that the combination of multimodal neuroimaging techniques has the potential not only to increase our understanding of structural disruptions in neuronal circuits associated with functional deficits in children with ADHD, but also to provide objective data that may assist the clinician in the day-to-day practice.
\end{abstract}

M. Angriman $(\bowtie)$

Child Neurology and Neurorehabilitation Unit, Central Hospital of Bolzano, Bohler Street, 5, Bolzano 39100, Italy

e-mail: marco.angriman@gmail.com

\section{A. Beggiato}

Department of Child and Adolescent Psychiatry, Assistance

Publique-Hôpitaux de Paris, Robert Debré Hospital, Paris, France

\section{A. Beggiato}

Human Genetics and Cognitive Functions Unit, Institut Pasteur, Paris, France

\footnotetext{
A. Beggiato

Child and Adolescent Neuropsychiatry Unit, Azienda Ospedaliera

Universitaria Integrata di Verona, Verona, Italy

S. Cortese

Cambridgeshire and Peterborough Foundation Trust,

Cambridge, UK
}

Keywords Attention-Deficit/Hyperactivity Disorder . Neuroimaging · Magnetic Resonance Imaging · fMRI · Structural imaging · Diffusion Tensor Imaging $\cdot$ Resting State · Graph Theory $\cdot$ Classification · Children

\section{Introduction}

According to the recently published Diagnostic and Statistical Manual of Mental Disorders, fifth edition, DSM-5 [1], attention-deficit/hyperactivity disorder (ADHD) is defined by a persistent and impairing pattern of inattention and/or hyperactivity/impulsivity. ADHD is a major public health issue. Its worldwide-pooled prevalence is estimated at about $5 \%$ [2]. It has been shown that impairing symptoms of ADHD may persist in adulthood in up to $65 \%$ of cases [3], with an estimated pooled prevalence of adulthood ADHD of $\sim 2.5 \%$ [4]. ADHD may be comorbid with a broad range of psychiatric disorders, such as oppositional defiant disorder, anxiety and disorders, and specific learning disorders [5], as well as with some medical conditions, such as sleep disorders [6-15], obesity [16-22] and, possibly, increased cardiovascular risk [23, 24], and iron deficiency $[25,26]$, at least in a subsample of cases.

In many countries, in particular in North America, the mainstay of treatment for ADHD is pharmacologic, with psychostimulants as first choice and non-psychostimulants as second option [27]. The efficacy of non-pharmacological approaches for ADHD core symptoms is still uncertain [28, 29], although some, such as behavioural interventions, may be effective for ADHD related conditions (i.e., oppositional behaviours).

The pathophysiology of the disorder is not yet entirely clear, possibly due to its heterogeneity. In past years, an increasing number of neuroimaging studies have been conducted to shed light on the neurobiology underlying ADHD: excellent recent narrative reviews (e.g. [30,31]) or systematic 
reviews/meta-analyses (e.g., [32••, 33, 34••]) are available on the neuroimaging of ADHD.

The senior author of this article focused on reviewing neuroimaging data collected from 2011 and 2012 [31]; here, we updated these papers providing an overview of the studies on the neuroimaging of childhood ADHD published in 2013.

\section{Method}

Although this is not intended to be a systematic review with a detailed quantitative appraisal of included studies, we searched in a broad range of electronic databases to increase the chances of find all pertinent published studies. Specifically, we searched the following databases: PubMed, Ovid PsychInfo, Ovide Medline, Web of Science, and EMBASE, limiting the search to 2013. We included only original empirical papers reporting studies in children. We excluded studies conducted in adults self-referred to adult ADHD clinics or studies conducted in children followed-up in adulthood (e.g., [35]). We used the same search terms of a recent review of the literature [30], as reported in Table 1. The search was updated on October 25, 2013. Figure 1 shows the results of our search. Table 2 reports the list of the retained papers. Further details on the search process/results are available upon request to the corresponding author.

Note: Each section starts with a background providing information on the specific MRI type discussed in the section as well as results from previous studies, followed by a summary of the key findings of papers published in the last year.

\section{Structural Imaging}

\section{Anatomical MRI}

\section{Starting Point}

Structural magnetic resonance imaging (MRI) is the primary imaging tool used to investigate brain anatomy. Frequently, used structural MRI measures include volume and density of the gray (GM) or white matter (WM) in the whole brain or in specific brain regions.

Using structural MRI, children with ADHD compared to controls have shown significant abnormalities in frontostriatal, fronto-temporo-parietal and fronto-cerebellar networks [34••]. These brain regions are known to guide cognitive control functions in inhibition, self-adjustment and goaldirected behaviors, which are impaired in ADHD. Structural MRI studies published in the last year have added important contributions to our current understanding of the anatomical alterations underpinning ADHD.
A group of recent studies has specifically focused on the assessment of cortical thickness. To fill the gap in our knowledge on the link between trajectories of cerebral cortical development in childhood and severity of ADHD in adulthood, Shaw et al. [36], performed a longitudinal study involving 92 participants with ADHD having childhood (mean 10.7 years, SD 3.3) and adult clinical assessments (mean 23.8 years, SD 4.3) with repeated brain MRIs, compared to 184 matched typically developing volunteers. ADHD persisted in $40 \%$ of subjects and symptom severity in adulthood was linked to cortical developmental trajectories: the rate of cortical thinning in medial prefrontal/cingulate and dorsolateral prefrontal cortex significantly correlate with increasing in inattentive symptoms. Interestingly, cortical thickening or minimal thinning (greater than $-0.007 \mathrm{~mm} /$ year) was found exclusively among individuals with remitted ADHD symptoms, so that the cortical thinning in remitted ADHD group tended to converge toward normal values, whereas the persistent ADHD group showed more fixed, nonprogressive deficits.

In another study, Almeida Montes et al. [37] compared whole-brain cortical-thickness (CT) in 59 medication-naïve male and female children, adolescents, and adults with ADHD and 62 matched controls. CT data revealed differences between ADHD and comparisons in the right hemisphere, which were significant in the posterior regions. Intriguingly, the CT differences were correlated with severity of ADHD but seemed to disappear with age: the percentage of the total right hemisphere cortical surface area occupied by these regions was higher in the child age group (2.29\%) compared with the adolescent $(1.02 \%)$ or adult $(0.19 \%)$ age groups. The authors speculated that increased $\mathrm{CT}$ in childhood is a compensatory mechanism for neuropsychological dysfunction and its progressive decrease is the result of altered peak pruning process during adolescence and adulthood; the effect of treatment on these compensatory mechanisms needs to further studies to be evaluated. Bledsoe et al. [38] aimed to analyze for the first time the impact of cortical thickness on functional outcome, correlating MRI parameters, neuropsychological testing and clinical severity in 32 children with ADHD and 15 healthy controls. The authors assessed structural cortical thickness for the bilateral rostral and caudal anterior cingulate cortex (ACC, implicated in behavioral error detection, impulsivity, and inhibitory control), inhibitory control with Stroop Inhibition test, and ADHD symptom severity with behavioral questionnaires filled out by parents and teachers. By means of regression model analysis, they showed that children with ADHD displayed significant cortical thinning in the right rostral ACC, unrelated to psychostimulant medication history, but nonsignificant thinning in the right caudal, left caudal, or left rostral ACC compared with healthy controls. Right rostral ACC thickness predicted also a part of the variance in behavioral questionnaire scores. No such relationship emerged between cortical thickness and inhibitory control performance. 
Table 1 Search terms for each database

\section{PUBMED (MEDLINE)}

Search terms:

(ADHD OR attention deficit hyperactivity disorder OR attention-deficit/hyperactivity disorder OR attention deficit disorder with hyperactivity OR hyperkinetic syndrome OR syndrome hyperkinetic OR hyperactivity disorder OR hyperactive child syndrome OR childhood hyperkinetic syndrome OR attention deficit disorder OR deficit disorder attention OR disorder attention deficit OR addh OR overactive child syndrome OR attention deficit hyperkinetic disorder OR hyperkinetic disorder OR attention deficit disorder hyperactivity OR child attention deficit disorder OR hyperkinetic syndrome childhood OR hyperkinesis) AND (Imaging OR neuroimaging OR magnetic resonance imaging OR MRI OR nuclear magnetic resonance imaging OR NMR imaging OR functional imaging OR functional magnetic imaging OR Functional MRI OR fMRI OR Positron emission tomography OR PET OR Tomography Positron-Emission OR positron emission tomographic scan OR positron tomography OR Positron emission tomography computer assisted OR Computer assisted positron emission tomography OR Positron emission computed tomography OR Single Photon Emission Compute Tomography OR CT Scan Single-Photon Emission OR Emission-Computed Tomography Single-Photon OR Tomography Single-Photon Emission-Computed OR SPECT OR computer assisted tomography single photon emission OR emission computer tomography single photon OR tomography emissioncomputed single-photon)

\section{OVID databases}

\section{PsycInfo}

Search terms:

(ADHD OR attention deficit hyperactivity disorder OR attention deficit disorder with hyperactivity OR hyperkinetic syndrome OR syndrome hyperkinetic OR hyperactivity disorder OR hyperactive child syndrome OR childhood hyperkinetic syndrome OR attention deficit disorder OR deficit disorder attention OR disorder attention deficit OR addh OR overactive child syndrome OR attention deficit hyperkinetic disorder OR hyperkinetic disorder OR attention deficit disorder hyperactivity OR child attention deficit disorder OR hyperkinetic syndrome childhood OR hyperkinesis OR Attention deficit disorder / OR ((attenti\$) adj3 (deficit\$ OR disorder\$ or hyperactiv\$ OR hyper?activ\$ OR adhd OR addh OR ad??hd)) OR ((hyperkin\$ OR hyper?kin\$) adj3 (deficit\$ OR disorder\$ OR hkd))) AND (Imaging OR neuroimaging OR magnetic resonance imaging OR MRI OR nuclear magnetic resonance imaging OR NMR imaging OR functional imaging OR functional magnetic imaging OR Functional MRI OR fMRI OR Positron emission tomography OR PET OR Tomography Positron-Emission OR positron emission tomographic scan OR positron tomography OR Positron emission tomography computer assisted OR Computer assisted positron emission tomography OR Positron emission computed tomography OR Single Photon Emission Compute Tomography OR CT Scan Single-Photon Emission OR Emission-Computed Tomography Single-Photon OR Tomography Single-Photon Emission-Computed OR SPECT OR computer assisted tomography single photon emission OR emission computer tomography single photon OR tomography emission-computed single-photon)

\section{Ovid Medline}

Search terms:

(ADHD OR attention deficit hyperactivity disorder OR attention deficit disorder with hyperactivity OR hyperkinetic syndrome OR syndrome hyperkinetic OR hyperactivity disorder OR hyperactive child syndrome OR childhood hyperkinetic syndrome OR attention deficit disorder OR deficit disorder attention OR disorder attention deficit OR addh OR overactive child syndrome OR attention deficit hyperkinetic disorder OR hyperkinetic disorder OR attention deficit disorder hyperactivity OR child attention deficit disorder OR hyperkinetic syndrome childhood OR hyperkinesis OR Attention deficit disorder / OR ((attenti\$) adj3 (deficit\$ OR disorder\$ or hyperactiv\$ OR hyper?activ\$ OR adhd OR addh OR ad??hd)) OR ((hyperkin\$ OR hyper?kin\$) adj3 (deficit\$ OR disorder\$ OR hkd))) AND (Imaging OR neuroimaging OR magnetic resonance imaging OR MRI OR nuclear magnetic resonance imaging OR NMR imaging OR functional imaging OR functional magnetic imaging OR Functional MRI OR fMRI OR Positron emission tomography OR PET OR Tomography Positron-Emission OR positron emission tomographic scan OR positron tomography OR Positron emission tomography computer assisted OR Computer assisted positron emission tomography OR Positron emission computed tomography OR Single Photon Emission Compute Tomography OR CT Scan Single-Photon Emission OR Emission-Computed Tomography Single-Photon OR Tomography Single-Photon Emission-Computed OR SPECT OR computer assisted tomography single photon emission OR emission computer tomography single photon OR tomography emission-computed single-photon)

\section{EMBASE + EMBASE CLASSIC}

Search terms:

(ADHD OR attention deficit hyperactivity disorder OR attention deficit disorder with hyperactivity OR hyperkinetic syndrome OR syndrome hyperkinetic OR hyperactivity disorder OR hyperactive child syndrome OR childhood hyperkinetic syndrome OR attention deficit disorder OR deficit disorder attention OR disorder attention deficit OR addh OR overactive child syndrome OR attention deficit hyperkinetic disorder OR hyperkinetic disorder OR attention deficit disorder hyperactivity OR child attention deficit disorder OR hyperkinetic syndrome childhood OR hyperkinesis OR Attention deficit disorder / OR ((attenti\$) adj3 (deficit\$ OR disorder\$ or hyperactiv\$ OR hyper?activ\$ OR adhd OR addh OR ad??hd)) OR ((hyperkin\$ OR hyper?kin\$) adj3 (deficit\$ OR disorder\$ OR hkd))) AND (Imaging OR neuroimaging OR magnetic resonance imaging OR MRI OR nuclear magnetic resonance imaging OR NMR imaging OR functional imaging OR functional magnetic imaging OR Functional MRI OR fMRI OR Positron emission tomography OR PET OR Tomography Positron-Emission OR positron emission tomographic scan OR positron tomography OR Positron emission tomography computer assisted OR Computer assisted positron emission tomography OR Positron emission computed tomography OR Single Photon Emission Compute Tomography OR CT Scan SinglePhoton Emission OR Emission-Computed Tomography Single-Photon OR Tomography Single-Photon Emission-Computed OR SPECT OR computer assisted tomography single photon emission OR emission computer tomography single photon OR tomography emission-computed single-photon) 
Table 1 (continued)

\section{WEB OF KNOWLEDGE}

(Web of science (science citation index expanded), Biological abstracts, Biosis, Food science and technology abstracts) Search terms:

(ADHD OR attention deficit hyperactivity disorder OR attention deficit disorder with hyperactivity OR hyperkinetic syndrome OR syndrome hyperkinetic OR hyperactivity disorder OR hyperactive child syndrome OR childhood hyperkinetic syndrome OR attention deficit disorder OR deficit disorder attention OR disorder attention deficit OR addh OR overactive child syndrome OR attention deficit hyperkinetic disorder OR hyperkinetic disorder OR attention deficit disorder hyperactivity OR child attention deficit disorder OR hyperkinetic syndrome childhood OR hyperkinesis) AND (Imaging OR neuroimaging OR magnetic resonance imaging OR MRI OR nuclear magnetic resonance imaging OR NMR imaging OR functional imaging OR functional magnetic imaging OR Functional MRI OR fMRI OR Positron emission tomography OR PET OR Tomography Positron-Emission OR positron emission tomographic scan OR positron tomography OR Positron emission tomography computer assisted OR Computer assisted positron emission tomography OR Positron emission computed tomography OR Single Photon Emission Compute Tomography OR CT Scan Single-Photon Emission OR Emission-Computed Tomography Single-Photon OR Tomography Single-Photon Emission-Computed OR SPECT OR computer assisted tomography single photon emission OR emission computer tomography single photon OR tomography emission-computed single-photon)

Besides cortical thickness, other anatomical aspects have been investigated. A study of cerebellar volume was conducted by Ivanov and collaborators [39] using surface morphometry technique in a cohort of 105 subjects aged $8-18$ years (46 ADHD versus 59 controls). Within-group analyses revealed a diminished volume in the cerebellar hemispheres and in the vermis (lobules IV, V and VI, left crus I and right crus II) correlated with symptoms severity, in ADHD group. Additionally, the treatment with stimulants medications was associated with a variation in regional cerebellar volume, supporting the theory of a compensatory morphological change due to stimulant effect.

In order to gain insight on possible different neural ADHD endophenotypes, Adisetiyo and coworkers [40 • carried out a whole-brain voxel-based analyses using diffusional kurtosis imaging on a cohort of 49 subjects aged $8-$ 18 years old (ADHD-mixed $n=22$ and typically developing controls $n=27)$. In the ADHD-pure subgroup $(n=11)$ compared to control group, a lack of age-dependent maturation and a greater complexity in grey and white matter micro-

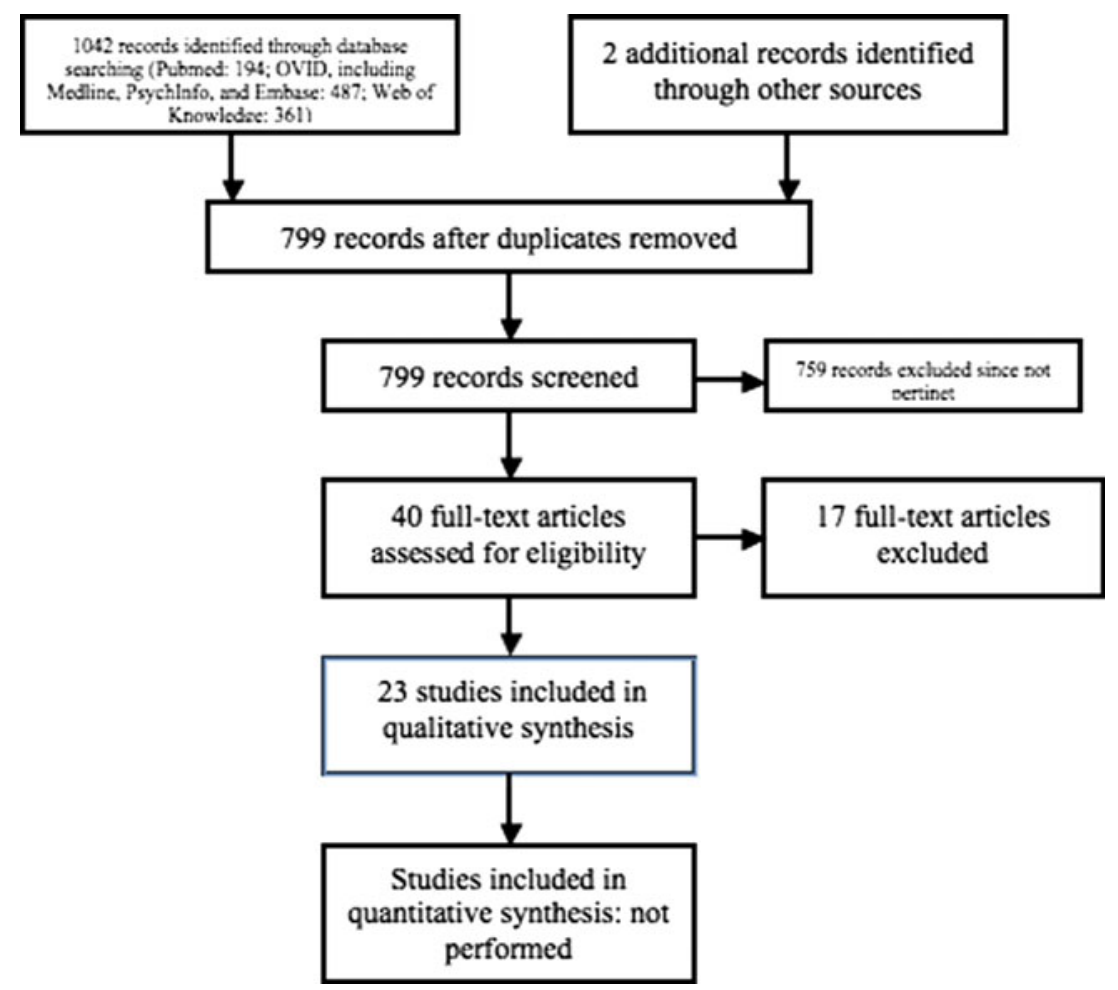

Fig. 1 Literature search results 
Table 2 List of included studies (in alphabetical order)

1. Adisetiyo V, Tabesh A, Di Martino A, et al. Attention-deficit/hyperactivity disorder without comorbidity is associated with distinct atypical patterns of cerebral microstructural development. Hum Brain Mapp. Aug 12013.

2. Almeida Montes LG, Prado Alcantara H, Martinez Garcia RB, De La Torre LB, Avila Acosta D, Duarte MG. Brain Cortical Thickness in ADHD: age, Sex, and Clinical Correlations. J Atten Disord. Nov 2013;17(8):641-654.

3. An L, Cao QJ, Sui MQ, et al. Local synchronization and amplitude of the fluctuation of spontaneous brain activity in attention-deficit/hyperactivity disorder: a resting-state fMRI study. Neurosci Bull. Oct 2013;29(5):603-613.

4. An L, Cao XH, Cao QJ, et al. Methylphenidate normalizes resting-state brain dysfunction in boys with attention deficit hyperactivity disorder. Neuropsychopharmacology. Jun 2013;38(7):1287-1295.

5. Bledsoe JC, Semrud-Clikeman M, Pliszka SR. Anterior cingulate cortex and symptom severity in attention-deficithyperactivity disorder. $J$ Abnorm Psychol. May 2013;122(2):558-565.

6. Cao Q, Shu N, An L, et al. Probabilistic diffusion tractography and graph theory analysis reveal abnormal white matter structural connectivity networks in drug-naive boys with attention deficit/hyperactivity disorder. $J$ Neurosci. Jun 26 2013;33(26):10676-10687.

7. Christakou A, Murphy CM, Chantiluke K, et al. Disorder-specific functional abnormalities during sustained attention in youth with Attention Deficit Hyperactivity Disorder (ADHD) and with autism. Mol Psychiatry. Feb 2013;18(2):236-244.

8. Chuang TC, Wu MT, Huang SP, Weng MJ, Yang P. Diffusion tensor imaging study of white matter fiber tracts in adolescent attention-deficit/ hyperactivity disorder. Psychiatry Res. Feb 28 2013;211(2):186-187.

9. Costa Dias TG, Wilson VB, Bathula DR, et al. Reward circuit connectivity relates to delay discounting in children with attention-deficit/hyperactivity disorder. Eur Neuropsychopharmacol. Jan 2013;23(1):33-45.

10. Cubillo A, Smith AB, Barrett N, et al. Drug-specific laterality effects on frontal lobe activation of atomoxetine and methylphenidate in attention deficit hyperactivity disorder boys during working memory. Psychol Med. Apr 19 2013:1-14.

11. Di Martino A, Zuo XN, Kelly C, et al. Shared and distinct intrinsic functional network centrality in autism and attention-deficit/hyperactivity disorder. Biol Psychiatry. Oct 15 2013;74(8):623-632.

12. Fair DA, Nigg JT, Iyer S, et al. Distinct neural signatures detected for ADHD subtypes after controlling for micro-movements in resting state functional connectivity MRI data. Front Syst Neurosci. 2012;6:80.

13. Hart H, Chantiluke K, Cubillo AI, et al. Pattern classification of response inhibition in ADHD: toward the development of neurobiological markers for ADHD. Hum Brain Mapp. Oct 112013.

14. Ivanov I, Murrough JW, Bansal R, Hao X, Peterson BS. Cerebellar Morphology and the Effects of Stimulant Medications in Youths with Attention Deficit-Hyperactivity Disorder. Neuropsychopharmacology. Sep 272013.

15. Lawrence KE, Levitt JG, Loo SK, et al. White matter microstructure in subjects with attention-deficit/hyperactivity disorder and their siblings. $J$ Am Acad Child Adolesc Psychiatry. Apr 2013;52(4):431-440 e434.

16. Lim L, Marquand A, Cubillo AA, et al. Disorder-specific predictive classification of adolescents with attention deficit hyperactivity disorder (ADHD) relative to autism using structural magnetic resonance imaging. PLoS One. 2013;8(5):e63660.

17. Lin HY, Gau SS, Huang-Gu SL, Shang CY, Wu YH, Tseng WY. Neural substrates of behavioral variability in attention deficit hyperactivity disorder: based on ex-Gaussian reaction time distribution and diffusion spectrum imaging tractography. Psychol Med. Aug 9 2013:1-14.

18. Posner J, Rauh V, Gruber A, Gat I, Wang Z, Peterson BS. Dissociable attentional and affective circuits in medication-naive children with attentiondeficit/hyperactivity disorder. Psychiatry Res. Jul 30 2013;213(1):24-30.

19. Shang CY, Wu YH, Gau SS, Tseng WY. Disturbed microstructural integrity of the frontostriatal fiber pathways and executive dysfunction in children with attention deficit hyperactivity disorder. Psychol Med. May 2013;43(5):1093-1107.

20. Shaw P, Malek M, Watson B, Greenstein D, de Rossi P, Sharp W. Trajectories of cerebral cortical development in childhood and adolescence and adult attention-deficit/hyperactivity disorder. Biol Psychiatry. Oct 15 2013;74(8):599-606.

21. Smith A, Cubillo A, Barrett N, et al. Neurofunctional effects of methylphenidate and atomoxetine in boys with attention-deficit/hyperactivity disorder during time discrimination. Biol Psychiatry. Oct 15 2013;74(8):615-622.

22. Tafazoli S, O’Neill J, Bejjani A, et al. 1H MRSI of middle frontal gyrus in pediatric ADHD. J Psychiatr Res. Apr 2013;47(4):505-512.

23. Wang S, Yang Y, Xing W, Chen J, Liu C, Luo X. Altered neural circuits related to sustained attention and executive control in children with ADHD: an event-related fMRI study. Clin Neurophysiol. Nov 2013;124(11):2181-2190.

structural organisation were found. The structures involved included the bilateral frontal and parietal lobes, insula, corpus callosum, right external and internal capsules. This finding led the authors to conclude that heterogeneous ADHD cohorts, with inclusion of ADHD-comorbid subsets, could mask specific neural phenotypes, possibly associated with specific neuroimaging markers of treatment response.
Key points

Trajectories of cerebral cortical development from childhood into adulthood are associated with the adult outcome of ADHD. In medial cortical regions, higher rates of cortical thinning are associated with higher levels of adult ADHD symptoms. Other data support the hypothesis that anatomical abnormalities in ADHD represent abnormal development rather than developmental delay. 
Diffusion Tensor Imaging (DTI)

\section{Starting Point}

Diffusion tensor imaging (DTI), a MRI method mapping the diffusion process of water, provides information about white matter connections and organization of fibers in the brain. Fractional anisotropy (FA), the most commonly used DTI metric, is an indirect index of the microstructure (axonal myelination, size, density and organization) of white matter. Recent DTI studies have elucidated important aspects on white matter (WM) microstructure alterations in ADHD

Lin et al. [41] found significantly reduced fractional anisotropy (suggesting miscrostructural alterations) in frontostriatal tracts, but not in the mid-cingulum bundles, in a sample of ADHD 28 youths with ADHD (17 combined type, 11 inattentive type; mean age 11.54 years, SD 2.30 years) compared to a strictly matched cohort of 28 comparisons (mean age 11.57 years, SD 2.75 years). Moreover, the authors investigated for the first time the correlation between these tracts and the intra-individual variability in reaction time, measured trough the Conners' continuous performance test go/no-go paradigm. Significant individual variability in reaction time was demonstrated in participants with ADHD and this finding was associated with cingulum bundle integrity in this group only. Shang et al. [42] compared anatomic structure of fronto-striatal regions and executive functions in 25 children with ADHD and 25 matched typically developing children, using psychiatric interviews, the Wechsler Intelligence Scale for Children (third edition), and the Cambridge Neuropsychological Test Automated Battery. The fronto-striatal tracts were reconstructed by diffusion spectrum imaging tractography and were subdivided into four functionally distinct segments. Children with ADHD displayed loss of the leftward asymmetry in the dorsolateral and medial prefrontal tracts, which was present in typically developing children. Furthermore, children with ADHD had significantly lower generalized fractional anisotropy of all the bilateral fronto-striatal fiber tracts and poorer performance in verbal and spatial working memory, set-shifting, sustained attention, cognitive inhibition and visuospatial planning. The symptom severity of ADHD and the executive functioning performance significantly correlated with microstructural integrity of the left orbitofrontal and ventrolateral tracts. A positive correlation between decrease of FA and neuropsychogical test results (Wechsler Intelligence Scale for Children and a non-verbal computerized battery of Mindstreams) was reported also by Chuang et al. [43] in a sample of 12 non-drug-naive ADHD and 14 control adolescent males. By means of a computational DTI technique know as tract-based spatial statistics (TBSS), the authors found significant lower FA in the white matter of middle cerebellar peduncle, corticospinal/corticobulbar tract, left inferior longitudinal fascicles, internal capsule, left optic radiation, bilateral splenium of corpus callosum, posterior corona radiata and left anterior corona radiata in the ADHD group compared to children without ADHD. The study results also indicated that global scores, executive function and attentional scores of the ADHD group were strongly predicted by the FA of the middle cerebellar peduncle, the internal capsule peduncle and the posterior corona radiata, respectively.

Another study has combined DTI derived techniques with sophisticated methods from graph theory to gain insight on complex structural alterations in WM networks in the whole brain ADHD. In this study, Cao et al. [44] examined WM connectivity in 30 drug naive boys with ADHD and 30 healthy controls. Both the ADHD and control groups showed an efficient small-world organization in the wholebrain WM networks, suggesting a balance between structurally segregated and integrated connectivity patterns. However, relative to controls, patients with ADHD exhibited decreased global efficiency and increased shortest path length, with the most pronounced efficiency decreases in the left parietal, frontal, and occipital cortices. Intriguingly, the ADHD group showed decreased structural connectivity in the prefrontal-dominant circuitry and increased connectivity in the orbitofrontal-striatal circuitry, and these changes significantly correlated with the inattention and hyperactivity/ impulsivity symptom scores, respectively.

Beside the assessment of WM alteration per se, recent investigation has also started to address the contribution of genetic and environmental factors to such abnormalities. Lawrence et al. [45] aimed to examine the contribution to ADHD familial liability to white matte microstructural alterations, performing DTI tractography to assess FA as well as other DTI metrics, including mean-diffusivity (MD), axial diffusivity (AD), and radial diffusivity (RD) in several a priori selected brain regions (regions of interest, ROIs) in children and adolescents with $\operatorname{ADHD}(\mathrm{n}=56)$, unaffected siblings of ADHD probands $(n=31)$, and healthy controls $(n=17)$. Subjects with ADHD showed significantly higher MD than controls in the anterior thalamic radiation, forceps minor, and superior longitudinal fasciculus, possibly reflecting impaired connectivity in executive function networks. Interestingly, medicated subjects with ADHD displayed significantly higher $\mathrm{MD}$ and $\mathrm{AD}$ in the forceps minor than non-medicated subjects with ADHD. More intriguingly, unaffected siblings of subjects with ADHD had similar differences in MD as subjects with ADHD, indicating a familial liability for the disorder. Overall, these data suggest that microstructural alterations in white matter reflect familial liability for ADHD and that MD may relate to structural abnormalities in a more sensitive way than FA. (MD is an index of myelin breakdown, altered cellular density and abnormal extracellular volumes). 
Key points

Recent data suggest that clinical symptomatology and executive functions correlate with integrity of the fronto-striatal tracts and support the role of dopamine in the pathophysiology of ADHD through neuromodulatory influences over fronto-striatal circuits. Microstructural alterations in white matter reflect familial liability for ADHD.

\section{Functional Imaging}

\section{Starting Point}

Functional MRI techniques provide a way to understand normal brain functions and to test for regional brain dysfunctions associated with disorders. Both task-based and restingstate fMRI have been frequently applied in children with ADHD, and have demonstrated atypical functional activations in the frontal, temporal, parietal lobes, and cerebellar regions.

Functional MRI (fMRI) studies have provided evidence for the fronto-striatal deficit hypothesis of ADHD in addition to providing evidence for wider deficits across other networks, including the default network [32••] and resting state functional MRI (rs-fMRI) adjunct data to the hypothesis of dysfunctional fronto-cerebellar circuits in ADHD. In the following sections, we highlight recent contributions to our understanding of functional alterations in ADHD, presenting separately task-based fMRI studies and resting state functional MRI investigations. We also present the results of a magnetic resonance imaging spectroscopy study.

\section{Task-based fMRI}

A recent study by Wang et al. [46] aimed to evaluate the temporal pattern of activation in fronto-cerebellar and parietal attentional networks during response execution in ADHD, comparing 28 medication-naïve children with ADHD aged 7-12 years and 31 healthy controls during a cued continuous performance task (AX-CPT) in three stimulus context conditions (go/no-go, Lure, which is a variant of the no-go condition requesting a larger and faster inhibitory response). Children with ADHD showed increased activation in the left middle frontal gyrus, bilateral middle temporal gyrus, left precuneus and right cerebellum posterior lobe under the Lure condition compared to the controls. In the Lure condition, in contrast to the no-go condition, an increased activation in the left inferior frontal gyrus, right medial frontal gyrus and right inferior parietal gyrus was observed in ADHD children.

An important line of research in the field aims to find ADHD-specific functional alterations that allow differentiating this condition form other child psychiatric disorders. Christakou et al. [47] conducted the first fMRI study comparing 20 boys with ADHD to 20 age- and IQ-matched ASD and 20 healthy boys during a parametrically modulated vigilance task with a progressively increasing load of sustained attention. ADHD and ASD boys showed significantly reduced activation relative to controls in bilateral striato-thalamic regions, left dorsolateral prefrontal cortex (DLPFC) and superior parietal cortex. Both groups also displayed significantly increased precuneus activation relative to controls. Precuneus activation was negatively correlated with the DLPFC activation in both groups, and this region was progressively more deactivated with increasing attention load in controls, but not in patients with ADHD and ASD, suggesting problems with deactivation of a task-related default mode network in these two disorders. Intriguingly, left DLPFC underactivation was significantly more pronounced in ADHD relative to ASD boys, which also was associated with sustained performance measures that were only impaired in ADHD patients. Boys with ASD, on the other hand, had disorder-specific enhanced cerebellar activation relative to both ADHD and control boys, presumably reflecting compensation for the fronto-striatal dysfunction.

Two studies have examined the pharmacological effects on brain activation in ADHD. In the first, Smith et al. [48] investigated both the effect of methylphenidate (MPH) on frontostriatal regions and the impact of atomoxetine (ATMX) on ventrolateral prefrontal networks. They assessed a sample of 20 ADHD adolescents by means of a randomized double-blind cross-over design after single doses of MPH, ATMX, and placebo during an fMRI time discrimination (TD) task. To assess the potential normalization effect of methylphenidate and atomoxetine, results were compared to brain activation in 20 healthy age-matched control subjects. Relative to controls, children on placebo showed TD deficits and reduced activation of ventrolateral prefrontal cortex (VLPFC)/insula, inferior frontal cortex, and supplementary motor area. Performance differences were normalized only by MPH, compared with both ATMX and placebo. Both medications, however, significantly upregulated activation in right VLPFC/insula in patients and also significantly upregulated and normalized right VLPFC underactivation in ADHD boys under placebo relative to control subjects, suggesting shared effects of psychostimulants and non-psychostimulants on a key prefrontal dysfunction during time discrimination tasks. In the second study from the same group, using a similar design, Cubillo et al. [49] performed three subsequent fMRIs in 20 medication-naive ADHD boys aged 10-17 years, compared with that of 20 age-matched healthy control boys, during a parametric verbal working memory (WM) task under three different pharmacological situations: single dose of MPH, of ATX or of placebo. Boys with ADHD showed impaired performance only under placebo and under high but not low WM load together with significant underactivation in the bilateral dorsolateral prefrontal cortex (DLPFC). On the contrary, both MPH and ATX normalized the performance deficits relative to controls: ATX significantly enhanced right DLPFC activation relative to MPH within 
patients; MPH, by contrast, upregulated the left inferior frontal cortex (IFC) both relative to placebo and ATX. These results suggest drug-specific laterality effects on prefrontal regions during working memory tasks, as both MPH and ATMX enhanced fronto-temporo-striatal activation in ADHD relative to control boys and deactivated the default-mode network, but ATX upregulated and normalized right DLPFC underactivation, while MPH upregulated left IFC activation.

Finally, we mention in this section a recent study based on a different technique, i.e. magnetic resonance spectroscopic imaging, which provides information about cellular (metabolic) activity. Tafazoli and colleagues [50] examined the bilateral middle frontal gyri in 13 children and adolescents with ADHD and 13 age- and sex-matched healthy controls. Neuropsychological assessment of attention was also carried out with the Trail Making Task in a subsample of the ADHD patients. It was found that, in the right middle frontal gyrus only, mean levels of $\mathrm{N}$-acetyl-aspartate $\mathrm{p} \mathrm{N}$-acetylaspartyl-glutamate (tNAA), creatine $\mathrm{p}$ phosphocreatine $(\mathrm{Cr})$, choline-compounds (Cho), and myo-inositol (mI) were significantly lower in the ADHD than in the control sample. Additionally, in patients with ADHD, lower right middle frontal $\mathrm{Cr}$ was associated with worse performance on Trails A and B (focused attention, concentration, set-shifting), while the opposite relationship held true for the control group on Trails B. Lower levels of these multiple species in the right middle frontal cortex may reflect osmotic adjustment to elevated prefrontal cortical perfusion in ADHD and/or a previously hypothesized defect in astrocytic production of lactate in ADHD resulting in decelerated energetic metabolism $(\mathrm{Cr})$, membrane synthesis (Cho, $\mathrm{mI})$, and acetylCoA substrate for NAA synthesis.

Key points

Task-based fMRI has revealed both commonalities and disorder specific under or hyperactivation ADHD and ASD, mirroring the clinical overlap and specificity of these two disorders. Task-based fMRI has also detected the common and specific neuronal correlates of methylphyenidate and atomoxetine, as well as drugspecific laterality effects on prefrontal regions.

Recent findings of neurometabolite depletion in the middle frontal gyrus suggest that healthy controls might consume less energy (i.e., get more efficient) as they improve at the task, whereas ADHD patients must expend more energy in order to perform the task better.

\section{Resting State MRI}

\section{Starting Point}

Whereas task based fMRI studies were the most commonly used and almost exclusive fMRI approach in the past decade, nowadays resting state MRI studies, due to the easiness in their implementation, are becoming more and more popular in child psychiatry neuroimaging. Studies published over the last year show that this holds true when considering specifically ADHD.

Recent resting state MRI studies (rs-MRI) of ADHD have addressed several issues. First, they have been used to better test theoretical models of ADHD. Costa Dias et al. [51] used the rs-MRI technique to examine how an imbalance in reward circuits, manifested with alterations in the nucleus accumbens (NAcc) connectivity, may relate to impulsive decision-making in ADHD. They compared 35 school-aged children with ADHD and 64 matched controls, and found that functional connectivity of NAcc was atypical in children with ADHD and that in ADHDcombined type, the connection between NAcc and left anterior and ventro-medial prefrontal cortex was significantly increased than in controls. Furthermore, the ADHDrelated increased connectivity between NAcc and the prefrontal cortex was associated with greater impulsivity measured in a delay discounting task. In the same sample, the NAcc also displayed strong negative connectivity with insula anterior cingulate cortex, middle temporal cortex and parietal regions, all implicated in impulse control. The authors speculate that atypical signaling of the NAcc to the prefrontal cortex in ADHD may lead to excessive approach and failure in estimating future consequences, leading to impulsive behavior.

In another study, Posner et al. [52] aimed to clarify the dual pathway model (i.e., the neural networks underlying two different types of neurocognitive function, executive attention, $\mathrm{EA}$, and emotional regulation, ER) which is supposed to underlie ADHD. Assessing a cohort of 22 medication naïve children with ADHD $(\mathrm{N}=22)$ and 20 controls by means of used resting-state functional connectivity MRI (rs-fc-MRI), the authors found alterations in connectivity in two systems: one underlying executive attention, including the ventral and dorsal attentional networks as well as the fronto-striatal system (correlated with executive attention deficits but not with emotional lability) and the other emotional regulation, composed of the ventral striatum, the sub-genual and orbitofrontal cortices, the amygdala, and the hippocampus (correlated with emotional lability but not with executive attention deficits). These findings suggest that ADHD pathophysiology cannot be subsumed under one discrete neural system or one neurocognitive domain but that at least two neurocognitive domains must be considered

Rs-MRI has also been employed to find commonalities and disorder specific alterations across psychopathology. Di Martino et al. [53 • ] compared patterns of connections in school-aged typically-developing, ASD and ADHD children, measured in resting state functional magnetic resonance imaging, voxelwise network centrality, functional connectivity 
metrics indexing local and global functional relationships across the entire brain connectome, in 56 children with ASD, 45 children with ADHD, and 50 typically-developing children. The authors found abnormalities in ADHD within the right caudate, pallidum, and putamen, as well as in the left postcentral cortex, and abnormalities referable to ASD in temporolimbic areas. When comparing children with ASD with and without ADHD-like comorbidity a shared neural signature between the ASD and the ADHD group (i.e., increased DC in right striatum and pallidum) was observed, which was absent in ASD without ADHD.

Similarly to what we have discussed for task-based MRI studies, rs-MRI has been used as well to assess the brain correlates of ADHD drugs. An et al. [54] investigated the acute effects of MPH in 23 boys with ADHD scanned twice, under either $10 \mathrm{mg}$ dose of MPH or placebo, in a randomized, cross-over, counterbalanced placebo controlled design and compared to 32 matched healthy controls. Seven ADHD boys participated in a follow up 8-week MPH treatment. Children under placebo, relative to controls, showed decreased ReHo (a measure of temporal synchronization in neighboring voxels in BOLD signals, reflective, according to the authors, coordination of combined regional neuronal activity subserving similar goals or representations) in bilateral superior frontal gyrus, as well as increased ReHo in bilateral sensorimotor cortex, right premotor area, left cuneus, right calcarine, and superior occipital gyri. MPH, relative to placebo significantly upregulated activity in left IFC, right OFC, and cerebellar vermis, and downregulated activity in right parietal and visual cortex that overlapped with the abnormally enhanced parieto-visual activity areas. When under MPH, ReHo differences between patients and controls were no longer observed. In the seven patients participating in the follow up study, decreased ReHo in right parietal cortex after the acute MPH was positively correlated with the decreased symptom scores after the 8week MPH treatment. The authors suggested that MPH may promote complex cognitive functions such as attention, timing, and working memory as well as ameliorate symptoms control in ADHD by augmenting both the regional activity and the functional connectivity in the frontoparieto-cerebellar circuit.

Another important area of investigation relates to the application of novel rs-MRI metrics and the assessment of the additional information that they can provide. Another study by An and colleagues [55], conducted in 23 medication-naïve boys with ADHD and 25 age-matched healthy male controls, underlined the complementary role of the amplitude of low frequency fluctuation (ALFF) approach and the ReHo analysis in measure of local spontaneous brain activity. Performing both ReHo and ALFF methods, this work displayed a hyperactivity of visual cortex in the ADHD group and a decreased local synchronization in the fronto-cingulo-parieto-cerebellar circuits.

\section{Key points}

Resting state MRI studies in ADHD are continuously evolving in terms of metrics that they adopt. These studies have confirmed that the pathophysiology and psychopathology underlying ADHD cannot be accounted for by a unique theoretical model; on the contrary, several pathways, including dysfunctions in executive functions, emotional regulation, and reward, are likely involved. Resting state MRI studies have detected commonalities and specificities in neuronal correlates between ADHD and other neurodevelopmental disorders, such as ASD. Resting state MRI studies have also shown that MPH may promote complex cognitive functions such as attention, timing, and working memory as well as ameliorate symptoms control in ADHD by augmenting both the regional activity and the functional connectivity in the fronto-parieto-cerebellar circuit.

\section{Prediction of Diagnosis of ADHD}

\section{Starting Point}

In the clinical practice, while diagnosing some cases of ADHD with clinical interviews is quite straightforward, the differential diagnosis of other cases may be very challenging. In recent years, neuroimaging studies have been conducted to provide information which is useful to diagnose a particular disorder at the individual patient level, rather than providing information that holds true at the group level (e.g., abnormalities found averaging the data from the scans conducted in a group of patients).

Neuroimaging studies aimed at predicting clinical diagnosis are increasingly conducted in ADHD. Lim et al. [56] applied Gaussian Process Classification (GPC) to grey matter volumetric data from 29 ADHD adolescents, 19 ASD patients and 29 healthy controls. The pattern of GM volumetric data correctly classified $79.3 \%$ of ADHD patients, and discriminating regions predictive of ADHD patients were most predominantly in earlier developing ventral brain regions relative to the more dorsal counterparts, such as bilateral ventrolateral, premotor and ventral temporal cortices, limbic regions including hippocampus, amygdala, hypothalamus, ventral striatum/ putamen, insula, posterior cingulate and brain stem. There was also a small cluster in the inferior vermis that had greater significance for ADHD, as opposed to the rest of the cerebellum. The positive and negative predictive values for the classifier were respectively $81.5 \%$ and $77.4 \%$.

In another recent study, Hart et al. [57•] applied Gaussian process classifiers to task-based (inhibition) fMRI in 30 adolescent ADHD and 30 age-matched healthy controls. The pattern of brain activation correctly classified up to $90 \%$ of patients and $63 \%$ of controls, achieving an overall classification accuracy of $77 \%$. Most predictive regions of ADHD 
were in earlier developing bilateral ventrolateral prefrontal, striatal, and temporo-parietal regions, which also correlated with symptom severity. The authors concluded that significant individual classification of ADHD patients can be achieved using whole brain pattern analysis of task-based fMRI inhibition data and that multivariate pattern recognition analyses of inhibition networks can provide objective diagnostic neuroimaging biomarkers of ADHD.

Using a different approach of support vector machine (SVM)-based multivariate pattern analysis (MVPA), Fair et al. [58] showed that functional connectivity patterns in individuals could differentiating the two most prominent ADHD subtypes (combined and inattentive). The application of graph-theory revealed that the ADHD-C and ADHD-I subtypes demonstrate unique patterns of atypical connectivity: in ADHD-C, midline default network components and insular cortex showed atypical connectivity; in contrast, dlPFC regions and cerebellum demonstrated atypical patterns in the ADHD-I group. The authors suggest that rs-fcMRI data can characterize individual patients with ADHD and detect distinctions underlying the clinical heterogeneity of ADHD at a neural level.

Key points

Neuroimaging studies start being used to predict the diagnosis of ADHD based on task-based and resting state MRI data. They also have been employed to differentiate patients based on specific neuronal correlates of the ADHD subtypes.

\section{Conclusion}

Neuroimaging studies of ADHD are increasingly used to test the theoretical models of its pathophysiology, providing data on the structural and functional alterations underlying the disorder. The combination of different imaging modalities will likely increase the reliability of the findings and lead to more rigorous pathophysiological models. In this way, neuroimaging may provide a pivotal tool toward the ambitious project set up by the NIMH referred as RdoC (research Domain criteria) aimed at re-conceptualizing the classification of mental illness based on behavioral dimensions grounded on neuroscience, in order to stratify broad phenotype in more specific constructs to improve diagnostic accuracy and response to treatment [59].

Classic neuroimaging studies in psychiatry have been criticized on the ground that they provide only neuronal correlates of disorders, confirming what it is already know by definition, i.e. that individuals affected by psychiatric disorders are "different" from healthy ones, and that are in a way "far" from the dayto-day clinical practice. The possibility to use neuroimaging data to predict diagnosis of ADHD might change this view, providing information that may have immediate impact on the clinical practice. Preliminary results from fMRI classification studies, if replicated across centers and scanners, may, therefore, set the ground for future clinical applications of neuroimaging, in particular as a complement to diagnose difficult cases. However, whereas such studies may provide useful data to help the clinician classify a subset of challenging cases, we believe that a major advance will be provided by neuroimaging studies aimed at predicting the functional outcome and the response to treatment. We look forward to these studies.

Conflict of Interest Marco Angriman, Anita Beggiato, and Samuele Cortese declare that they have no conflict of interest.

Human and Animal Rights and Informed Consent This article does not contain any studies with human or animal subjects performed by any of the authors.

\section{References}

Papers of particular interest, published recently, have been highlighted as:

- Of importance

•- Of major importance

1. American Psychiatric Association: Diagnostic and Statistical Manual of Mental Disorder, Fifth Edition. ed American Psychiatric Publishing, 2013.

2. Polanczyk G, de Lima MS, Horta BL, Biederman J, Rohde LA. The worldwide prevalence of ADHD: a systematic review and metaregression analysis. Am J Psychiatry. 2007;164:942-8.

3. Faraone SV, Biederman J, Mick E. The age-dependent decline of attention deficit hyperactivity disorder: a meta-analysis of followup studies. Psychol Med. 2006;36:159-65.

4. Simon V, Czobor P, Balint S, Meszaros A, Bitter I. Prevalence and correlates of adult attention-deficit hyperactivity disorder: metaanalysis. Br J Psychiatry. 2009;194:204-11.

5. Biederman J, Faraone SV. Attention-deficit hyperactivity disorder. Lancet. 2005;366:237-48.

6. Cortese S, Konofal E, Lecendreux M, Arnulf I, Mouren MC, Darra F, et al. Restless legs syndrome and attention-deficit/hyperactivity disorder: a review of the literature. Sleep. 2005;28:1007-13.

7. Cortese S, Konofal E, Yateman N, Mouren MC, Lecendreux M. Sleep and alertness in children with attention-deficit/hyperactivity disorder: a systematic review of the literature. Sleep. 2006;29:504-11.

8. Cortese S, Lecendreux M, Mouren MC, Konofal E. ADHD and insomnia. J Am Acad Child Adolesc Psychiatry. 2006;45:384-5.

9. Cortese S, Konofal E, Lecendreux M, Mouren MC, Bernardina BD. Restless legs syndrome triggered by heart surgery. Pediatr Neurol. 2006;35:223-6.

10. Cortese S, Maffeis C, Konofal E, Lecendreux M, Comencini E, Angriman M, et al. Parent reports of sleep/alertness problems and ADHD symptoms in a sample of obese adolescents. J Psychosom Res. 2007;63:587-90.

11. Cortese S, Konofal E, Dalla BB, Mouren MC, Lecendreux M. Does excessive daytime sleepiness contribute to explaining the association between obesity and ADHD symptoms? Med Hypotheses. 2008;70:12-6. 
12. Cortese S, Konofal E, Lecendreux M. Effectiveness of ropinirole for RLS and depressive symptoms in an 11-year-old girl. Sleep Med. 2009;10:259-61.

13. Cortese S, Brown TE, Corkum P, Gruber R, O'Brien LM, Stein M, et al. Assessment and management of sleep problems in youths with attention-deficit/hyperactivity disorder. J Am Acad Child Adolesc Psychiatry. 2013;52:784-96.

14. Konofal E, Lecendreux M, Cortese S. Sleep and ADHD. Sleep Med. 2010;11:652-8.

15. Lecendreux M, Cortese S. Sleep problems associated with ADHD: a review of current therapeutic options and recommendations for the future. Expert Rev Neurother. 2007;7:1799-806.

16. Cortese S, Bernardina BD, Mouren MC. Attention-deficit/hyperactivity disorder (ADHD) and binge eating. Nutr Rev. 2007;65:404-11.

17. Cortese S, Isnard P, Frelut ML, Michel G, Quantin L, Guedeney A et al. Association between symptoms of attention-deficit/hyperactivity disorder and bulimic behaviors in a clinical sample of severely obese adolescents. Int J Obes (Lond). 2007;31:340-6.

18. Cortese S, Angriman M. Attention-deficit/hyperactivity disorder and obesity: moving to the next research generation. Pediatrics. 2008;122:1155-6.

19. Cortese S, Angriman M, Maffeis C, Isnard P, Konofal E, Lecendreux M, et al. Attention-deficit/hyperactivity disorder (ADHD) and obesity: a systematic review of the literature. Crit Rev Food Sci Nutr. 2008;48:524-37.

20. Cortese S, Morcillo PC. Comorbidity between ADHD and obesity: exploring shared mechanisms and clinical implications. Postgrad Med. 2010;122:88-96.

21. Cortese S. Ramos Olazagasti MA, Klein RG, Castellanos FX, Proal E, Mannuzza S: Obesity in men with childhood ADHD: a 33-year controlled, prospective, follow-up study. Pediatrics. 2013;131:e1731-8.

22. Cortese S, Faraone SV, Bernardi S, Wang S, Blanco C. Adult attention-deficit hyperactivity disorder and obesity: epidemiological study. Br J Psychiatry. 2013;203:24-34.

23. Angriman M, Bruni O, Cortese S. Does Restless Legs Syndrome increase cardiovascular risk in Attention-Deficit/Hyperactivity Disorder? Med Hypotheses. 2013;80:39-42.

24. Parisi P, Villa MP, Donfrancesco R, Miano S, Paolino MC, Cortese S. Could treatment of iron deficiency both improve ADHD and reduce cardiovascular risk during treatment with ADHD drugs? Med Hypotheses. 2012;79:246-9.

25. Cortese S, Azoulay R, Castellanos FX, Chalard F, Lecendreux M, Chechin D, et al. Brain iron levels in attention-deficit/hyperactivity disorder: a pilot MRI study. World J Biol Psychiatry. 2012;13:223-31.

26. Donfrancesco R, Parisi P, Vanacore N, Martines F, Sargentini V, Cortese S: Iron and ADHD: Time to Move Beyond Serum Ferritin Levels. J Atten Disord 2012.

27. Pliszka S. Practice parameter for the assessment and treatment of children and adolescents with attention-deficit/hyperactivity disorder. J Am Acad Child Adolesc Psychiatry. 2007;46:894-921.

28. Sonuga-Barke E, Brandeis D, Cortese S, Daley D, Danckaerts M, Dopfner M, et al. Response to Chronis-Tuscano et al. and Arns and Strehl. Am J Psychiatry. 2013;170:800-2.

29. Sonuga-Barke EJ, Brandeis D, Cortese S, Daley D, Ferrin M, Holtmann M, et al. Nonpharmacological interventions for ADHD: systematic review and meta-analyses of randomized controlled trials of dietary and psychological treatments. Am J Psychiatry. 2013;170:275-89.

30. Cortese S, Castellanos FX. Neuroimaging of attention-deficit/hyperactivity disorder: current neuroscience-informed perspectives for clinicians. Curr Psychiatry Rep. 2012;14:568-78.

31. Cortese S. The neurobiology and genetics of Attention-Deficit/ Hyperactivity Disorder (ADHD): what every clinician should know. Eur J Paediatr Neurol. 2012;16:422-33.

32.• Cortese S, Kelly C, Chabernaud C, Proal E, Di MA, Milham MP, et al. Toward Systems Neuroscience of ADHD: A Meta-Analysis of
55 fMRI Studies. Am J Psychiatry. 2012;169:1038-55. A comprehensive meta-analysis of $f M R I$ studies in ADHD interepreting results in the light of system neuroscience.

33. Cortese S, Castellanos FX, Eickhoff SB: What are neuroimaging meta-analytic procedures? Epidemiol Psychiatr Sci 2013;1-3.

34.• Nakao T, Radua J, Rubia K, Mataix-Cols D. Gray matter volume abnormalities in ADHD: voxel-based meta-analysis exploring the effects of age and stimulant medication. Am J Psychiatry. 2011;168:1154-63. The most recent meta-anlysis of structural MRI studie sin ADHD.

35. Cortese S, Imperati D, Zhou J, Proal E, Klein RG, Mannuzza S, et al. White matter alterations at 33-year follow-up in adults with childhood attention-deficit/hyperactivity disorder. Biol Psychiatry. 2013;74:591-8.

36. Shaw P, Malek M, Watson B, Greenstein D, de Rossi P, Sharp W. Trajectories of cerebral cortical development in childhood and adolescence and adult attention-deficit/hyperactivity disorder. Biol Psychiatry. 2013;74(8):599-606.

37. Almeida Montes LG, Prado AH, Martinez Garcia RB, De la Torre LB, Avila AD, Duarte MG. Brain Cortical Thickness in ADHD: Age, Sex, and Clinical Correlations. J Atten Disord. 2013;17:641-54.

38. Bledsoe JC, Semrud-Clikeman M, Pliszka SR. Anterior cingulate cortex and symptom severity in attention-deficit/hyperactivity disorder. J Abnorm Psychol. 2013;122:558-65.

39. Ivanov I, Murrough JW, Bansal R, Hao X, Peterson BS: Cerebellar Morphology and the Effects of Stimulant Medications in Youths with Attention Deficit-Hyperactivity Disorder. Neuropsychopharmacology 2013

40. Adisetiyo V, Tabesh A, Di MA, Falangola MF, Castellanos FX, Jensen JH, Helpern JA: Attention-deficit/hyperactivity disorder without comorbidity is associated with distinct atypical patterns of cerebral microstructural development. Hum Brain Mapp 2013. An interesting study exploring different microstructural markers of $A D H D$ in relation to ADHD subtypes

41. Lin HY, Gau SS, Huang-Gu SL, Shang CY, Wu YH, Tseng WY: Neural substrates of behavioral variability in attention deficit hyperactivity disorder: based on ex-Gaussian reaction time distribution and diffusion spectrum imaging tractography. Psychol Med 2013;1-14.

42. Shang CY, Wu YH, Gau SS, Tseng WY. Disturbed microstructural integrity of the frontostriatal fiber pathways and executive dysfunction in children with attention deficit hyperactivity disorder. Psychol Med. 2013;43:1093-107.

43. Chuang TC, Wu MT, Huang SP, Weng MJ, Yang P. Diffusion tensor imaging study of white matter fiber tracts in adolescent attention-deficit/hyperactivity disorder. Psychiatry Res. 2013;211: 186-7.

44. Cao Q, Shu N, An L, Wang P, Sun L, Xia MR, et al. Probabilistic diffusion tractography and graph theory analysis reveal abnormal white matter structural connectivity networks in drug-naive boys with attention deficit/hyperactivity disorder. J Neurosci. 2013;33: 10676-87.

45. Lawrence KE, Levitt JG, Loo SK, Ly R, Yee V, O'Neill J, et al. White matter microstructure in subjects with attention-deficit/hyperactivity disorder and their siblings. J Am Acad Child Adolesc Psychiatry. 2013;52:431-40.

46. Wang S, Yang Y, Xing W, Chen J, Liu C, Luo X. Altered neural circuits related to sustained attention and executive control in children with ADHD: An event-related fMRI study. Clin Neurophysiol. 2013;124:2181-90.

47. Christakou A, Murphy CM, Chantiluke K, Cubillo AI, Smith AB, Giampietro V, et al. Disorder-specific functional abnormalities during sustained attention in youth with Attention Deficit Hyperactivity Disorder (ADHD) and with autism. Mol Psychiatry. 2013;18:236-44. 
48. Smith A, Cubillo A, Barrett N, Giampietro V, Simmons A, Brammer $\mathrm{M}$, et al. Neurofunctional effects of methylphenidate and atomoxetine in boys with attention-deficit/hyperactivity disorder during time discrimination. Biol Psychiatry. 2013;74:615-22.

49. Cubillo A, Smith AB, Barrett N, Giampietro V, Brammer M, Simmons A, Rubia K: Drug-specific laterality effects on frontal lobe activation of atomoxetine and methylphenidate in attention deficit hyperactivity disorder boys during working memory. Psychol Med 2013;1-14.

50. Tafazoli S, O'Neill J, Bejjani A, Ly R, Salamon N, McCracken JT, et al. 1H MRSI of middle frontal gyrus in pediatric ADHD. J Psychiatr Res. 2013;47:505-12.

51. Costa Dias TG, Wilson VB, Bathula DR, Iyer SP, Mills KL, Thurlow BL, et al. Reward circuit connectivity relates to delay discounting in children with attention-deficit/hyperactivity disorder. Eur Neuropsychopharmacol. 2013;23:33-45.

52. Posner J, Rauh V, Gruber A, Gat I, Wang Z, Peterson BS. Dissociable attentional and affective circuits in medication-naive children with attention-deficit/hyperactivity disorder. Psychiatry Res. 2013;213: 24-30.

53.• Di Martino A, Zuo XN, Kelly C, Grzadzinski R, Mennes M, Schvarcz A, et al. Shared and distinct intrinsic functional network centrality in autism and attention-deficit/hyperactivity disorder. Biol Psychiatry. 2013;74:623-32. A rigorously conducted study applying novel resting state metrics to find commonalities and specificties between ADHD and ASD.
54. An L, Cao XH, Cao QJ, Sun L, Yang L, Zou QH, et al. Methylphenidate normalizes resting-state brain dysfunction in boys with attention deficit hyperactivity disorder. Neuropsychopharmacology. 2013;38:1287-95.

55. An L, Cao QJ, Sui MQ, Sun L, Zou QH, Zang YF, et al. Local synchronization and amplitude of the fluctuation of spontaneous brain activity in attention-deficit/hyperactivity disorder: a restingstate fMRI study. Neurosci Bull. 2013;29:603-13.

56. Lim L, Marquand A, Cubillo AA, Smith AB, Chantiluke K, Simmons A, et al. Disorder-specific predictive classification of adolescents with attention deficit hyperactivity disorder (ADHD) relative to autism using structural magnetic resonance imaging. PLoS One. 2013;8:e63660.

57. Hart H, Chantiluke K, Cubillo AI, Smith AB, Simmons A, Brammer MJ, Marquand AF, Rubia K: Pattern classification of response inhibition in ADHD: Toward the development of neurobiological markers for ADHD. Hum Brain Mapp 2013. The first study using Gaussian process classifiers $(G P C)$ to predict the diagnosis of $A D H D$

58. Fair DA, Nigg JT, Iyer S, Bathula D, Mills KL, Dosenbach NU, et al. Distinct neural signatures detected for ADHD subtypes after controlling for micro-movements in resting state functional connectivity MRI data. Front Syst Neurosci. 2012;6:80.

59. Insel T, Cuthbert B, Garvey M, Heinssen R, Pine DS, Quinn K, et al. Research Domain Criteria(RDoC): toward a new classification framework for research on mental disorders. Am J psychautry. 2010;167:748-51. 